\title{
POSE ESTIMATION FOR SKELETON DETECTION
}

\author{
Ms Rashmi Raghavenda Patil \\ Dept. of Computer Science and Engineering \\ KLE's Dr. M S Sheshgiri College of Engineering and \\ Technology Belagavi, Karnataka, India
}

\author{
Prof Sushma V Chaugule \\ Dept. of Computer Science and Engineering \\ KLE's Dr. M S Sheshgiri College of Engineering \\ and Technology. Belagavi, Karnataka, India
}

\author{
Dr. V S Malemath \\ Department of computer science and engineering \\ KLE's Dr M S Sheshgiri College of Engineering and \\ Technology Belagavi, Karnataka, India
}

\begin{abstract}
This thesis is based on efficiently detecting the joints and coordinates of the person and limbs are highlighted with color dots and connecting them with drawn lines. The architecture will give briefs about activation functions and GPUs available on the used interfacing platform. Activation Rectified linear unit which used for computer vision to find body joint coordinates. This method uses COCO model for key points challenge and method works for all static image given to the process.
\end{abstract}

Index Terms - Deep learning, graphical processing units, COCO Model

\section{INTRODUCTION}

A Human Pose Skeleton shows the direction of an individual in a graphical arrangement. Basically, it is a lot of directions that can be associated with depict the posture of the individual. Every co-ordinate in the skeleton is known as a section (or a joint, or a keypoint). A legitimate association between two sections is known as a couple (or an appendage). Note that, not all part mixes offer ascent to substantial sets.

Knowing the direction of an individual opens roads for a few genuine applications, some of which are talked about towards the finish line. A few ways to deal with Human Pose Estimation were presented throughout the years. The most punctual (and slowest) techniques normally assessing the posture of a solitary individual in a picture which just had one individual in the first place. These techniques frequently distinguish the individual parts first, trailed by shaping associations between them to make the posture.

\section{LITERATURE SURVEY}

These are the papers which briefs about the investigation and work done by researchers which gives many dimensions and thought process to the current research work and project.
In Go, R., \& Aoki, Y[1] proposes the DeepPosebased posture estimation framework that is adaptable with the difference in bouncing box run for top-see pictures. This work present Bounding-box Curriculum Learning (BCL) and Recurrent Pose Estimation (RPE). BCL is a taking in strategy of CNN enlivened from Curriculum Learning. It demonstrate the impact of proposed strategies contrasted with ordinary scholarly CNN-put together posture estimator with respect to our unique top-see dataset.

In Poirson, P. \& et al [2] presents for applications in navigation and robotics technology, evaluating the 3D posture of items is as significant as recognition. Numerous ways to deal with posture estimation depend on recognizing or following parts or key points. In this work author expand on an ongoing best in class convolutional arrange for slidingwindow location to give identification and unpleasant posture estimation in a solitary shot, without middle phases of recognizing parts or introductory bouncing boxes. The way to the engineering is a profound convolutional organize where scores for the nearness of an item classification, the balance for its area, and the posture are altogether evaluated on a framework of areas in the picture.

In Chu, X \& et al [3] proposes to join convolutional neural systems with a multi-setting consideration instrument into a start to finish structure for human posture estimation. It receives stacked hourglass systems to produce consideration maps from highlights at different goals with different semantics. The Conditional Random Field (CRF) is used to show the relationships among neighboring districts in the consideration map. Consequently model can concentrate on various granularity from neighborhood remarkable locales to worldwide semantic predictable spaces. Also, structure novel Hourglass Residual Units (HRUs) to expand the open field of the system.

In Cao, Z. \& et al [4] Gives a way to deal with productively identify the $2 \mathrm{D}$ posture of different individuals in a picture. The methodology utilizes a nonparametric portrayal, which alludes to as Part Affinity Fields (PAFs), to figure out how to identify body parts with people in the picture. The 


\section{International Journal of Engineering Applied Sciences and Technology, 2019 \\ Vol. 4, Issue 4, ISSN No. 2455-2143, Pages 192-196 \\ Published Online August 2019 in IJEAST (http://www.ijeast.com)}

engineering is intended to together adapt part areas and their affiliation through two parts of the equivalent successive forecast process. This technology puts first in the debut $\mathrm{COCO}$ 2016 keypoints challenge, and fundamentally surpasses the past best in class result on the MPII Multi-Person benchmark, both in execution and proficiency.

In Tome, D., Russell, C., (2017) \&et al [5], brought together definition for the issue of 3D human posture estimation from a RGB picture that reasons mutually about 2D joint estimation and $3 \mathrm{D}$ pose reconstruction to improve the two errands. It can adopt a coordinated strategy that breakers probabilistic learning of $3 \mathrm{D}$ human posture with a multiarrange $\mathrm{CNN}$ engineering and uses the information of conceivable $3 \mathrm{D}$ milestone areas to refine the scan for better $2 \mathrm{D}$ areas.

In Liu, Y., Xu, Y. (2018) \& et al [6], pose estimation is a significant research subject in the field of computer vision and machine learning. Here centers around the condition art of 2-D human posture estimation strategies dependent on deep learning. As indicated by the neural system structure, these techniques are named single CNN strategy, Multi-arrange CNN technique, Multi-branch CNN strategy, Recurrent Neural Network (RNN) technique and Generative Adversarial Networks (GAN) strategy.

In Ai, B., Zhou, Y., Yu, Y. (2017) 4et al [7] This work fuses structure information into Convolutional Neural Networks (CNNs) for verbalized human posture estimation from a single still picture. It is unique in relation to existing techniques utilizing highlights from CNNs to display the tree structure, here it legitimately utilizes the structure present before guide the learning of $\mathrm{CNN}$. In the first place, it presents a deep CNN with successfully captures the holistic content of images. Second, limb loss is utilized as intermediate supervision of $\mathrm{CNN}$ to gain proficiency with the connections of joints. Just utilizing one phase and with no unpredictable post handling, this technique accomplishes condition of stateof-art results on both FLIC and LSP benchmarks.

\section{PROPOSED SYSTEM}

Normally, these strategies are not especially valuable in some genuine situations where pictures contain various individuals.

\section{A. Multi-Person Pose Estimation}

Multi-Person present estimation is more troublesome than the single individual case as the area and the quantity of individuals in a picture are obscure. Commonly, we can handle the above issue utilizing one of two methodologies:

- The basic methodology is to consolidate an individual identifier first, trailed by evaluating the parts and after that computing the posture for every individual. This strategy is known as the top-down methodology.

- Another approach is to identify all parts in the picture (for example portions of each individual), trailed by partner/gathering parts having a place with unmistakable people. This technique is known as the base up methodology.

- Normally, the top-down methodology is simpler to execute than the bottom up methodology as including an individual finder is a lot less complex than including partner/gathering calculations. It is difficult to pass judgment on which approach has better execution by and large as it truly comes down to which among the individual indicator and partner/gathering calculations is better.

This work mainly concentrate on multi-individual human posture estimation utilizing deep learning methods. In the following area, it audit a portion of the prominent topdown and bottom up methodologies for the equivalent.

\section{B. Pre-processing}

Skeleton detection is a crucial technology for apps that include image as part of the scene. It is need of the time to keep track of as many people that appear in the image. Now, with image processing combined with AI, confidence matric and heat maps generates a dots and lines joining those dots.

CNN Image classification accepts an input as a picture with any extension and processes and manipulate it for specific classification. Vision or AI sees input as picture to exhibit of pixels and it relies upon the picture goals. In view of the image resolution.

Height $x$ Width $x$ Dimension

This design accepts input as a RGB image of size $\mathrm{h} x$ $\mathrm{w}$, and output will be an array of matrices. This matrices consist of key points which are highlighted at the points where joints are found.

1. The initial 10 layers are utilized to make highlight maps for the image.

2. Branch multi-organize $\mathrm{CNN}$ is utilized where

The first branch predicts a lot of 2D Confidence Maps (S) of body joints areas (for example elbow, knee and so forth.). A Confidence Map is a picture which has a high color intensity at areas where the probability of a specific body joint is high. For instance, the Confidence Map for the Left Shoulder is appeared in Figure 4.2 underneath. It has high qualities at all areas where the there is a left shoulder.

3. For the 18 point model, the initial 19 frameworks of the yield relate to the Confidence Maps.

\section{Extraction}

PyTorch is explicitly ML library for the programming language Python, in light of the Torch library, utilized for applications, for example, image classification. This library is used for extraction and classification.

PyTorch gives two state highlights: 
- $\quad$ Tensor computing (like NumPy) with solid increasing speed through graphics processing units (GPU)

- Deep neural systems based on a tape-based auto diff framework

In this project GPU is used for processing and to remove noise, Gaussian filter is used. Gaussian filter changes the input signal with convolution using Gaussian function.

\section{Matrices:}

Percentage of Correct Parts : It gives the measurement of detection rate of limbs, where limb is viewed as identified if the separation between the two anticipated joint areas and the genuine limb joint areas is all things considered portion of the appendage length.

- Percent of Detected Joints: A joint is viewed as distinguished if the separation between the anticipated and the genuine joint is inside a specific division of the middle breadth. By fluctuating this portion, detection rates are acquired for differing degrees of confinement exactness

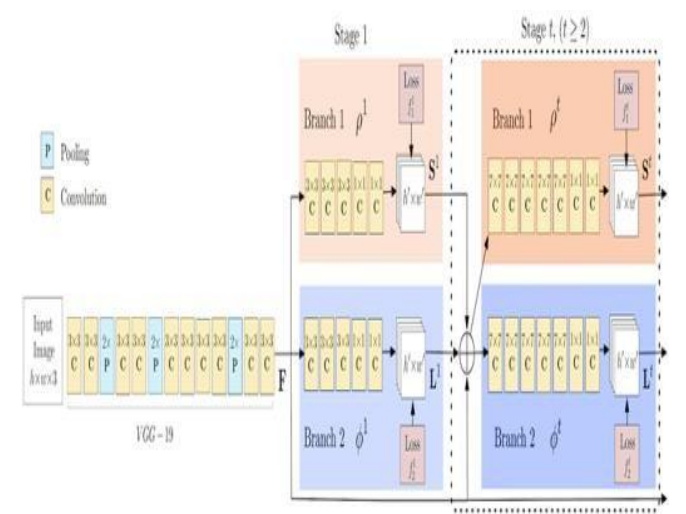

Fig 3.1 Neural network with layers

This system gives the skeleton estimation of the body. Which takes input as a image and gives estimated image and first it create a dots around joints after that it joins those joints. It finds connection in the specified sequence, center 29 is in the position 15 for the given image then get the middle joints heatmap correspondence values. Defining visualize colors for the input to get desired output.GPU is used to for processing in colaborotary.

\section{IMPLEMENATION}

\section{A. Gaussian filter}

The Gaussian filter alone will obscure edges and decrease differentiate. The Median filter is a non-linear filter that is most regularly utilized as a basic method to decrease noise in a picture.

$$
g(x)=\sqrt{\frac{a}{\pi}} \cdot e^{-a \cdot x^{2}}
$$

\section{B. Non Linearity $(\operatorname{ReLU})$}

Abbreviation of ReLU is Rectified Linear Unit for a non-linear operation.

$$
f(x)=\max (0, x) .
$$

Purpose of ReLU's is tell about non-linearity in ConvNet till the data might want ConvNet to train would be non-negative linear values.

There are 17 joints which are going to be identified by this method.

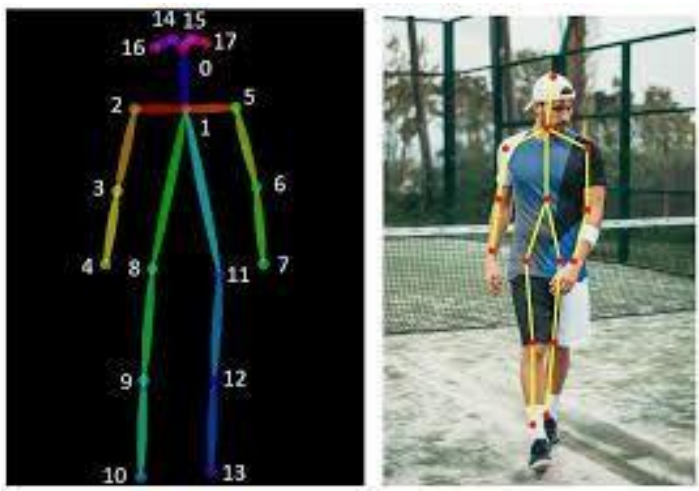

Fig 4.1 Body joints

\section{COCO Model}

$\mathrm{COCO}$ is an enormous image dataset intended for object detection, segmentation, person's joint keypoints identification, stuff segmentation, and caption generation. This package gives Matlab, Python, and Lua APIs that helps with stacking, parsing, and envisioning the comments in COCO. http://cocodataset.org/for more data on COCO, including for the information, paper, and instructional exercises. The precise configuration of the explanations is additionally depicted on the COCO site. The Matlab and Python APIs are finished, the Lua API gives just essential usefulness.

Notwithstanding this API, if it's not too much trouble download both the COCO pictures and comments so as to run the demos and utilize the API. Both are accessible on the venture site.

\section{EXPERMENTALRESULTS}

After image loading it generates the image with heatmap and confidence matrices with the following frame outline. This gives graph of the image. 
CPu tines: user $36 \mathrm{~ns}, 5 \mathrm{ys}: 8 \mathrm{~ns}$, total: $36 \mathrm{~ns}$
hall tine: $35.2 \mathrm{~ns}$

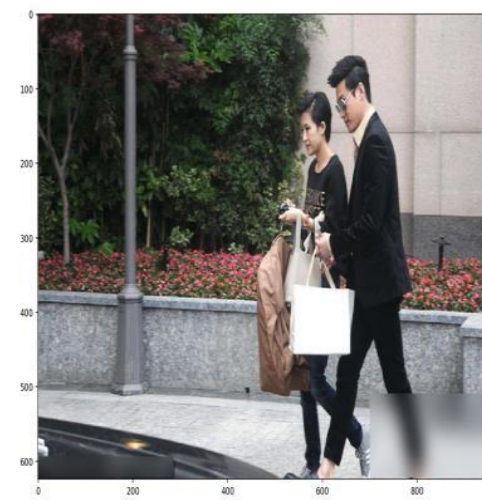

Fig 5.1 input image after loading

Next coco model is loaded in environment.it gives estimated values of conv2D which are sequential. It has parameters as shown in the figure i.e kernel size, stride, padding.

After loading image it generates the following image which has 2 image partition. First section shows the dots on the image and second part shows the dots joined by line.

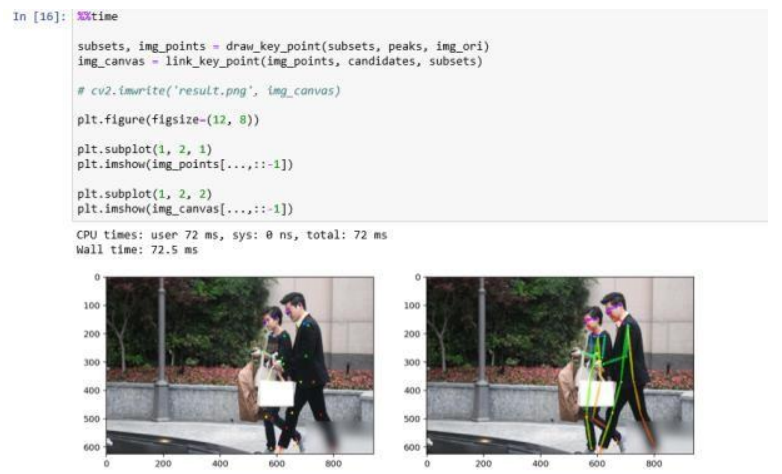

Fig 5.2 Result.png which is generated by the system
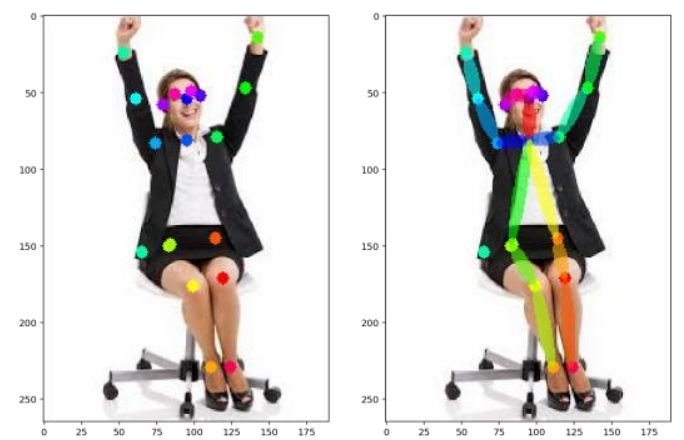

Fig 5.3 A person with sitting pose
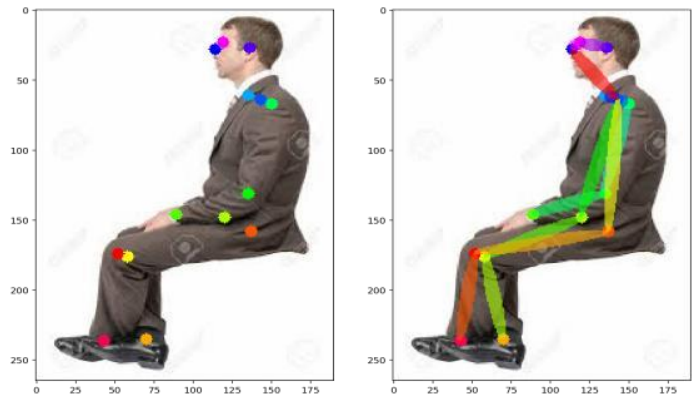

Fig 5.4 person sitting with side pose

\section{CONCLUSION}

This project work introduces a single person and also of multiple individual joint detection for person's pose estimation in clicked images or else downloaded images. This methods establishes the advantages of structure which depends on pose demands that categorized at various limb areas in the input picture which are filtered and extracted using rectified linear unit. The proposed methods stands out by the environments in image and noise disturbance. It works well for all the images.

\section{FUTURE WORK}

In this work the dots of the joints are detected and joined by the color lines, as a future wok, project can be extended for live tracking of person and body movement of the person. It can be enhanced for simultaneously track and detect poses of many people in video and as well as in image which statically given a input.

This method can be also build on part affinity field representation for static images and can give architecture which can encode and predict spatio temporal affinity fields (STAF). Such methods will be useful to give live tracking observations and online inference so to achieve well-structured and deterministic bottom-up approach which is run time method to the number of individual within the given input image.

After classification records can stored for future analysis and the dataset will be recorded. When any new data input comes it tries to match and retrieve from the database.

\section{REFERANCE}

[1] Go, R., \& Aoki, Y. (2016). Flexible top-view human pose estimation for detection system via CNN. 2016 IEEE 5 th Global Conference on

Consumer

Electronics. doi:10.1109/gcce.2016.7800406

[2] Poirson, P., Ammirato, P., Fu, C.-Y., Liu, W., Kosecka, J., \& Berg, A. C. (2016). Fast Single Shot Detection and Pose 
Estimation. 2016 Fourth International Conference on 3D Vision (3DV).

[3] Chu, X., Yang, W., Ouyang, W., Ma, C., Yuille, A. L., \& Wang, X. (2017). Multi-context Attention for Human Pose Estimation. 2017 IEEE Conference on Computer Vision and Pattern Recognition (CVPR).

[4] Cao, Z., Simon, T., Wei, S.-E., \& Sheikh, Y. (2017). Realtime Multi-person 2D Pose Estimation Using Part Affinity Fields. 2017 IEEE Conference on Computer Vision and Pattern Recognition (CVPR).

[5] Tome, D., Russell, C., \& Agapito, L. (2017). Lifting from the Deep: Convolutional 3D Pose Estimation from a Single Image. 2017 IEEE Conference on Computer Vision and Pattern Recognition (CVPR).

[6] Liu, Y., Xu, Y., \& Li, S. (2018). 2-D Human Pose Estimation from Images Based on Deep Learning: A Review. 2018 2nd IEEE Advanced Information Management,Communicates,Electronic and Automation Control Conference (IMCEC).

[7] Ai, B., Zhou, Y., Yu, Y., \& Du, S. (2017). Human Pose Estimation Using Deep Structure Guided Learning. 2017 IEEE Winter Conference on Applications of Computer Vision (WACV).

[8] Xiaoqin Zhang, Changcheng Li, Weiming $\mathrm{Hu}$, Xiaofeng Tong, Maybank, S., \& Yimin Zhang. (2014). Human Pose Estimation and Tracking via Parsing a Tree Structure Based Human Model. IEEE Transactions on Systems, Man, and Cybernetics: Systems, 44(5), 580-592. [9] Ouyang, W., Chu, X., \& Wang, X. (2014). Multisource Deep Learning for Human Pose Estimation. 2014 IEEE Conference on Computer Vision and Pattern Recognition.

[10] Vosoughi, S., \& Amer, M. A. (2018). Deep 3D Human Pose Estimation Under Partial Body Presence. 2018 25th IEEE International Conference on Image Processing (ICIP). 